\title{
A Resource List Management Tool for Undergraduate Students Based on Linked Open Data Principles
}

\author{
Chris Clarke \\ Talis Group Limited, \\ Knights Court, Birmingham Business Park, B37 7YB \\ chris.clarke@talis.com
}

\begin{abstract}
Resource List Management tools help educators create and publish lists of resources relevant to students undertaking a particular module, course or assignment. Traditional approaches to online delivery of these lists have been limited by lack of interoperability and integration with other campus systems, poor take-up by instructors and brittle linking strategies which break as institutions shift suppliers for e-content from year to year. In this paper we present a Resource List Management tool that uses Semantic Web technologies and Linked Data principles to overcome these limitations and improve the interoperability of data contained within such systems.
\end{abstract}

\section{Introduction}

In this paper we present a Resource List Management system for university students based on Semantic Web technologies [1] and Linked Data principles [2], and in active use at a number of UK universities.

Resource Lists are collections of text books, journal articles, Web pages and/or audio visual content intended to be companions for students to degree courses, modules or assignments. These lists, defined by course instructors, were traditionally provided to the student as a paper handout comprising a list of citations, the intention being for the student to visit the campus library or bookshop to obtain the listed resources.

Over the past 10 to 15 years, these materials have increasingly become available over the Web, with publishers and content owners moving to an e-delivery model for both monographs and serials. Instructors have been making increasing use of blogging, podcasting and multimedia learning objects in their syllabuses. Resource List Management (RLM) tools emerged over this period, moving paper lists to online electronic signposting solutions.

While this transition has created many benefits by improving access to published lists, current RLM solutions have a number of limitations, particularly regarding interoperability of and wider access to the data they contain. In Section 2 we will discuss these limitations in more detail. Section 3 presents the system from the user perspective and outlines its usage context at the pilot sites (University of Plymouth and University of Sussex). In Section 4 we describe our technical approach based on the Semantic Web technology stack [1] and Linked Data principles [2], and detail many of the design decisions taken when developing the system. We conclude the paper in Section 5 with final observations and directions for future work. 


\section{Limitations of Traditional RLM Tools}

\subsection{Limited Interoperability of Resource List Data}

Historically, the interoperability of data between publishing platforms, the University library catalogue, the University Virtual Learning Environment (VLE) and RLM tools themselves has been poor. RLM tools have often been no more than electronic replicas of the paper based list, storing details about the lists and resources themselves within proprietary database schemas, accessible only by a HTML or other userorientated interface, with no open APIs available for integration.

Assuming that a new solution can provide better integration with other systems and the wider web, the issue of licensing data provided by APIs is oft-overlooked. We recognise the importance of being specific in how you wish others to use data in section 4.4.

\subsection{Few Opportunities for Collaboration or Personal Contributions}

RLM tools have not traditionally encouraged, or even allowed for, contributions from the majority of the users of the system (the students). Instead the emphasis has been on instructors creating a static list that is then used in read-only mode by the students. There has been little attempt to allow students to add valuable annotations to lists. Examples would include communicating whether they have found an item useful in attaining their learning goals; annotating resources with study notes; or forming new collections of resources for a particular essay or group exercise.

Not only do these restrictions contradict the notion of the Web as a read/write medium, recently popularised by trends often labelled as Web2.0 [3], but it also contradicts the view that learning could or should be approached as a collaborative process [4].

From the perspective of instructors, the task of discovering new or revised content to update existing or create new lists has been largely unaddressed by existing systems beyond offering a simple bookmarking system or search interface over the local university library catalogue. With hundreds if not thousands of instructors worldwide facing the task of creating, for example, an introductory physics resource list there is great potential for a more collaborative solution.

\subsection{Complexity in Access Mechanisms}

While the transition to electronic resources has improved the access landscape to some extent, it has also led to the situation where a single journal subscription can be fulfilled by numerous different publisher or aggregator platforms. This is known as the appropriate copy problem [5]. As an institution shifts subscription agents from year to year, the landscape of access rights a university has purchased becomes increasingly complex. Links to e-content harvested by the instructor into the RLM to may function this year, but expire or move to a different platform the next.

Institutional link resolver solutions, such as SFX from ExLibris ${ }^{1}$, go some way to solving these issues. However, in our experience, such library-biased solutions often represent a poor experience for the student and are potentially forgotten about by the instructor when assembling lists. Instead of searching the resolver and harvest-

${ }^{1}$ http://www.exlibris.co.il/category/SFXOverview 
ing the resource's resolver page, many instructors naturally harvest direct from the source web page to which the resolver points.

\subsection{Stock Management}

Traditional RLM tools have overcome a number of the limitations of paper-based resource lists, most notably in the area of stock management. Web-based lists that link to the resources in the university's library catalogue can be used to give advance warning to library acquisition staff that particular resources will be in high demand during certain periods and that stock levels may need to be increased.

However, existing systems are primitive and brittle in their approach, relying on catalogue database identifiers to link to resources, and fail to identify identical or equivalent items available in the catalogue such as duplicate copies or subsequent editions of a work. This results in sub-optimal algorithms for predicting both usage and availability for a resource.

\section{The Talis Resource List Management System}

In partnership with 15 UK and Eire universities (the focus group), we embarked on a project to replace Talis' existing RLM tool and seek to address some of these issues through the use of Semantic Web technologies.

The system, known as Talis Aspire ${ }^{2}$, was launched at the University of Plymouth, one of the existing focus group partners, in September 2008, initially with just 1000 students. Throughout the autumn semester, Plymouth have been increasing adoption of the system with the aim of giving access to all 22000 students in 2009. During January 2009, the University of Sussex joined the pilot, increasing access to around 40000 users in total.

Before we discuss our technical approach we will give an overview of the application from the perspective of our two primary user groups - students and instructors.

\subsection{The Instructor's User Experience}

The lists themselves can be constructed using sections (see figure 1), as defined in the Resource List ontology, to group resources according to topics, time periods, or importance. For example an instructor may group items into a time-based section (e.g. "Lecture 1" ) or use a topic-based (e.g. "Accounting for Intangibles") arrangement. This, along with any class notes the instructors add, both add extra context for the student and enhance the semantics of the list, and indicate intended uses for a group of resources. This makes the lists much more semantically-rich, and provides high quality contextual data for the future construction of recommendation services (see section 5).

The resources themselves are gathered into the system by a process known as harvesting. Using a bookmarklet, and in a similar pattern to sites such as del.icio.us ${ }^{3}$,

\footnotetext{
${ }^{2} \mathrm{http}: / /$ www.talis.com/aspire

${ }^{3}$ http://del.icio.us
} 
instructors can harvest resources from a multitude of web sites, including the library catalogue. These are placed in a holding area known as "My Library" where they can be further annotated, tagged or commented upon. Later, when the instructor is making changes to an existing list, or creating a new list, the library appears on the interface and resources can be dragged and dropped onto the appropriate place on the list.

\section{University of Plymouth Home My Resources Sign in Feedback WIN $£ 50$}

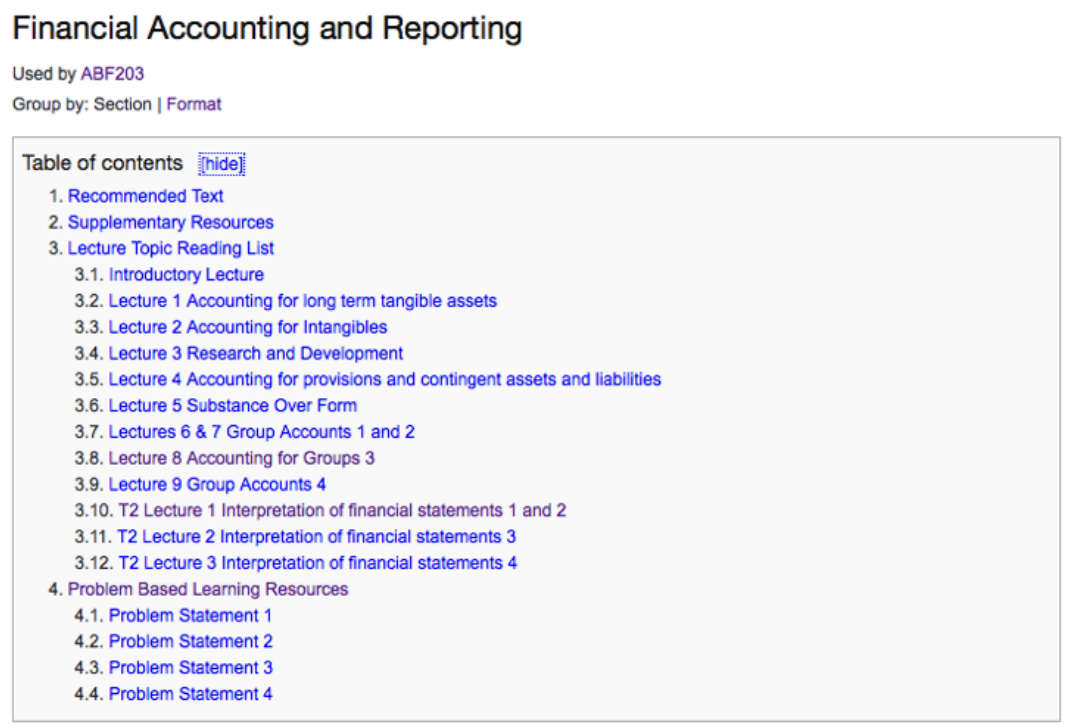

(93 items)

Recommended Text (2 items)

Financial accounting and reporting - Elliott, Barry., Elliott, Jamie. , 2008.

Get this item | Availability, buying options and notes

The 12th edition of Elliott and Elliott is the recommended text to buy for this module.

Financial Accounting and Reporting, 12/E - Pearson Education EMA Catalogue

Get this item | Availability, buying options and notes

This is the companion website and student resources for the 12 th edition

Fig. 1. Outline view of the list, with the table of contents in open mode. The blue box represents a section containing two item references.

The difference between the system and most bookmarking services is that the system attempts to identify the resource the page is describing, rather than just recording the location of the page itself. A series of recognisers are applied to the page content to determine the particulars of the item being harvested. Recognisers use a variety of techniques, including querying of other sources, based on facts gleaned from the page content, or 
evaluating microformat-style markup such as $\mathrm{COinS}^{4}$ or embedded RDF to pull metadata from the page. Only if a resource cannot be identified by automatic means is the user prompted to supply metadata and classify the type of resource being harvested.

\subsection{The Student's User Experience}

The student can interact with the resources within the lists in a number of ways. For physical items, the system will pull in stock availability from the local library catalogue, as well as offer a number of buying options. However, there are a number of functions designed not only to improve the experience for the student, but also increase the richness of the data (see figure 2).

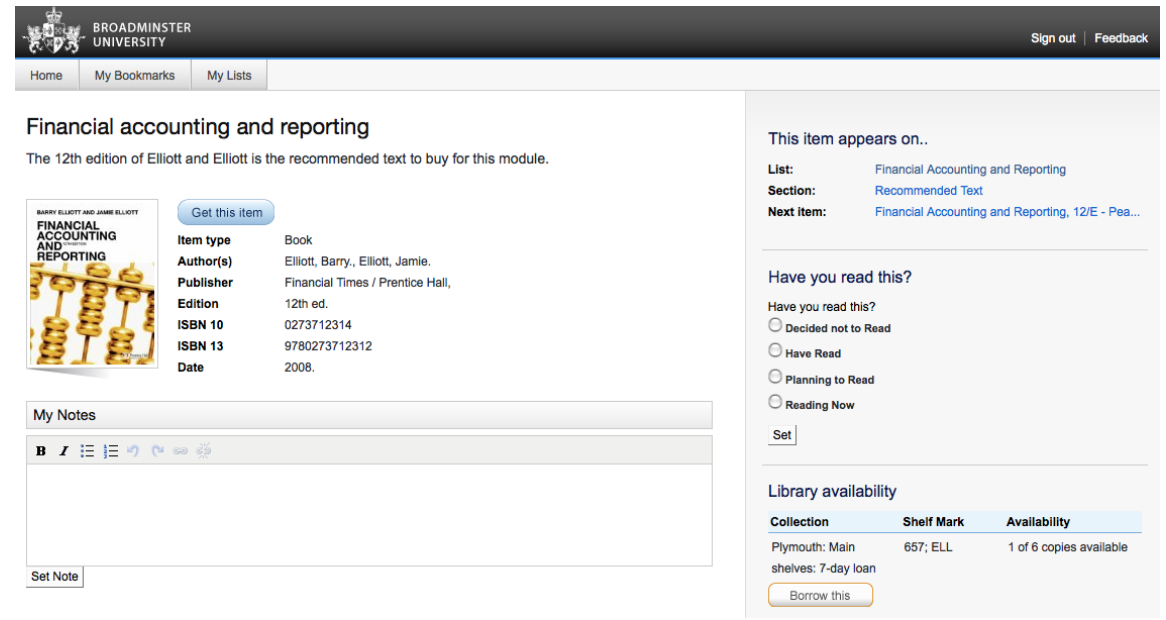

Fig. 2. The student experience (individual item view) showing the harvested metadata, personal study notes, read status and library availability. This screenshot is from the demonstration system, depicting the fictitious Broadminster University.

For example, using the Resource List ontology [6], the system allows the student to annotate each item on a list, augmenting the instructor's class notes with their own, and allowing to categorize each of the resources by intention (e.g. "Planning to read", "Reading now"). The student can then use the tool to manage their library of resources, typically running into the thousands, much more effectively. These intentions are stored as RDF triples in the application backend (invisibly to the user) and could be used as the basis for other applications. For example, a book loan service could be created, connecting level 3 students who own a core text with incoming level 2 students that are seeking to obtain it. Additionally, these intentions will be used in a forthcoming version of the system to enhance feedback given to instructors as to the popularity of the resources, by overlaying the raw usage statistics gathered from web server logs with the intentions of the students in the class.

\footnotetext{
${ }^{4}$ http://ocoins.info/
} 


\section{Technical Approach}

As mentioned above, the system is built on Semantic Web technologies (RDF, SPARQL, RDFa) and Linked Data principles ${ }^{5}$, which set out a recipe for linking and publishing data on the web via de-referenceable URIs. It is our view that this approach affords the following advantages in the context of this application:

- Unify the descriptions of resources using existing ontologies

- Allow these descriptions to be merged and matched with other bibliographic repositories

- Use ontologies designed for appropriation to describe the relationships between these resources and how they fit into the institutional hierarchy

- Improve the interoperability of the data using linked open data principles

- Encourage semantic enrichment of the data by students and instructors to enable context-aware recommendation functionality

The application is built in PHP, utilising the $\mathrm{ARC}^{6}$ - and Moriarty ${ }^{7}$ libraries, and stores its data natively in RDF.

\subsection{RDF Data Storage}

The system uses the Talis Platform [7] to store, index and query across its RDF data. The application makes use of a group of distinct stores to separate graphs with different concerns and levels of privacy (see figure 3). For example, publicly linkable and discoverable data is stored in a different store from private user content or details, such as preferences, passwords or private study notes. Data from these stores can be merged within the system at run time when required. Using this approach encourages re-use of the public data by other applications. Full SPARQL and search capabilities can be offered externally without exposing sensitive or private data.

As previously described, instructors harvest metadata about resources into the system using an intelligent bookmarklet. Once obtained, the metadata about these resources is stored in the instructor's library as RDF using the Bibliographic Ontology [8] increasing the interoperability of the harvested data with other systems and workflows. Bibliographic Ontology is already in use by a number of other groups, including Zotero ${ }^{8}$ a tool to help collect research sources, and the LIBRIS project ${ }^{9}$ at the National Library of Sweden which is a linked open data online library catalogue.

The system employs a mechanism of trusted sources to improve the quality of harvested metadata. Rather than rely solely on the metadata provided by the source web page as bookmarked by the user, the system scans the source for identifiers such as DOIs ${ }^{10}$ or ISBNs. These are then used to query trusted sources such as the

\footnotetext{
${ }^{5} \mathrm{http}: / /$ linkeddata.org/

${ }^{6} \mathrm{http}: / /$ arc.semsol.org/

${ }^{7}$ http://n2.talis.com/wiki/Moriarty

${ }^{8} \mathrm{http}: / /$ www.zotero.org

${ }^{9} \mathrm{http}: / /$ libris.kb.se/?language $=\mathrm{en}$

${ }^{10} \mathrm{http} / / /$ www.doi.org/
} 


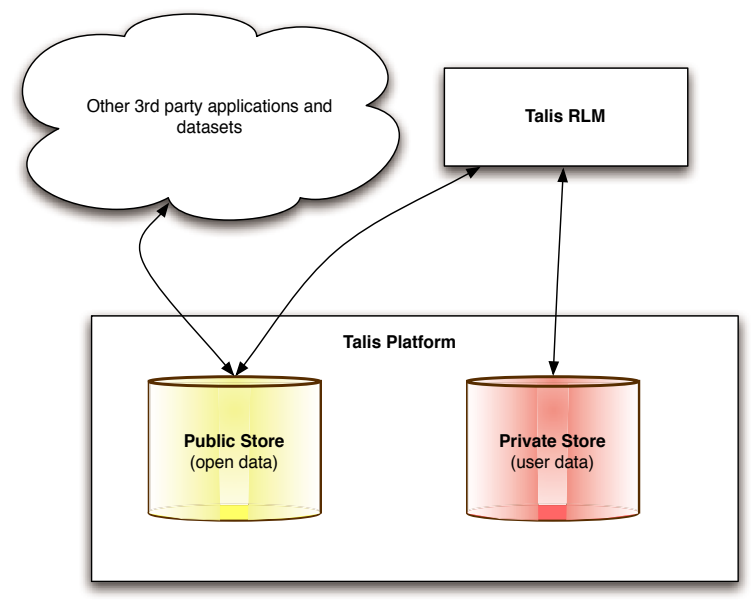

Fig. 3. Partitioning of data stores, with publicly linkable data in the store on the left, and private data, only available to the application, in the store on the right

institution's library catalogue, or the publisher's record for a journal article. A logical extension to this in the near future is to use a common identifier to mint a URI according to a known algorithm to produce a de-referenceable URI in the linked open data cloud, and retrieve the metadata from there. It would be easy to mint a URI referencing a resource in the RDF Book Mashup ${ }^{11}$ from an ISBN, for example, and follow the URI to discover metadata about the resource.

By storing metadata about the resource being described, rather than the page describing it, more resilient strategies can be employed to ensure content links do not break if the library decides in future to change supplier. Much of this more sophisticated logic is simply invoked by the user by clicking the "Get this item" button (see figure 1). The philosophy of this feature is to get the user as close to the resource as possible. To improve the user experience, content such as eBooks or journal articles that are available on-line are in-lined in the page without linking to an external location, in the style of YouTube ${ }^{12}$ and the Google Books ${ }^{13}$ services.

The interface to build or edit lists (see figure 4) uses a WYSIWYG metaphor implemented in Javascript to allow the user to drag and drop resources and edit data quickly, without the need to round trip back to the server on completion of each operation. Behind the scenes the actions of moving, adding, grouping or editing resources create RDFa [9] markup on the page. When the user has finished editing, they hit a save button which serialises the RDFa model in the page into an RDF/XML model which is submitted back to the server to be persisted to disk.

Adoption of this approach has significantly reduced complexity in the persistence logic of the application. The user effectively builds the RDF representation of the list

\footnotetext{
${ }^{11} \mathrm{http} / / / \mathrm{www} 4$.wiwiss.fu-berlin.de/bizer/bookmashup/

$12 \mathrm{http} / / /$ www.youtube.com

${ }^{13} \mathrm{http} / / /$ books.google.com
} 
themselves on the client, and submits the new model along with the model as it appeared before changes were applied to the server at the end of the editing session. The system simply compares the two models to calculate a delta of changes, and these are submitted to the appropriate store using the Changeset protocol [10].

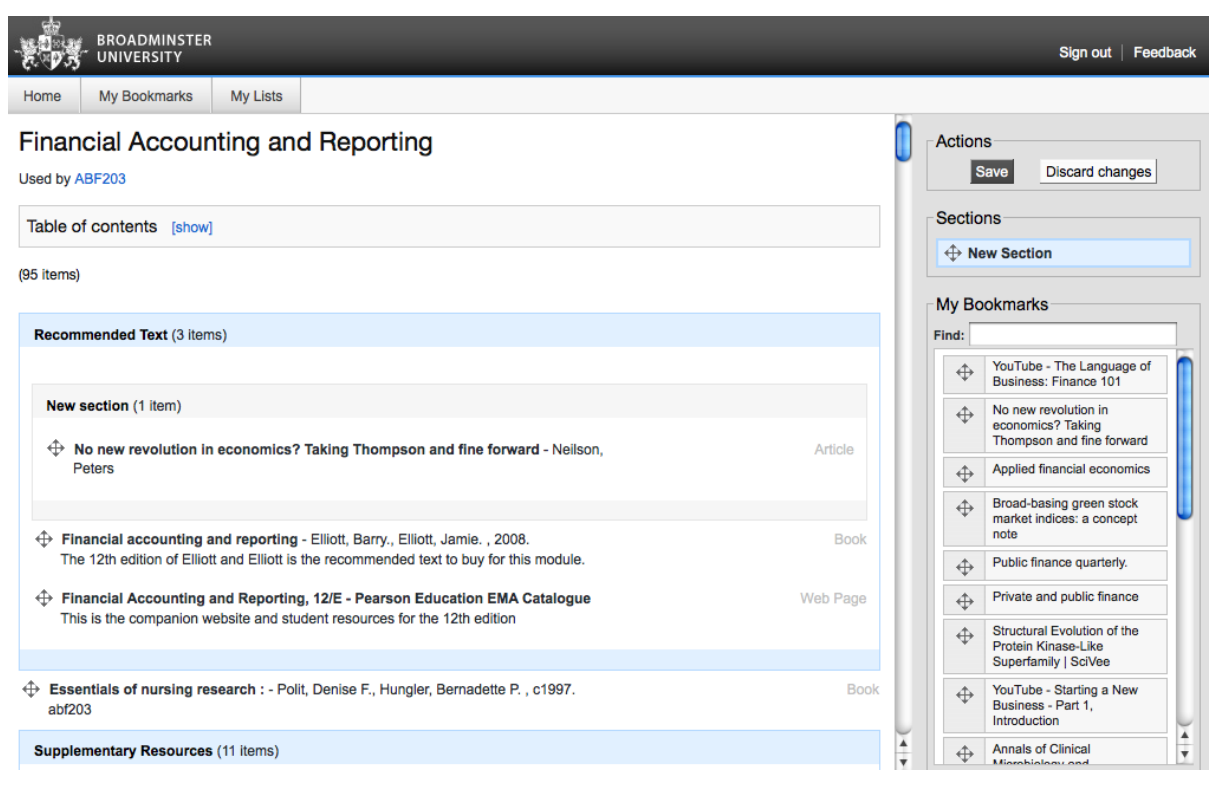

Fig. 4. The list editing. Harvested references can be dragged from the right and dropped onto the list on the left. References already on the list can be dragged and re-ordered.

\subsection{Vocabularies and Ontologies Used}

As well as re-using existing ontologies such as FOAF [11], SKOS [12], Biblio Ontology [8] and Dublin Core [13], we developed and published two new ontologies as part of the project. The Resource List Ontology [6] underpins the semantics of the relationships between resources and their intended uses. The Academic Institution Internal Structure ontology (AIISO) [14] describes the courses, modules, departments and schools that make up an institution, which was required to enable instructors to link lists to the relevant module or course, to enable students to find lists.

\subsection{Interoperability of Data}

As well as standardising the description of resources by using ontologies in use by other groups, the RLM tool encourages re-use and linking of data to other external sets by adhering to linked open data principles [15]. This means that not only did the design of the data have to fit the immediate use cases of the user, but also unknown use cases beyond the system boundary. This is referred to as designing for appropriation.

Unlike many web applications, the system is architected to use a noun-based URL structure to describe entities, rather than a verb-based approach to describe operations 
(see table 1). This works with the mechanisms that underpin the web, rather than against them, and focuses on describing resources via dereferencable URIs rather than describing operations [16]. This approach lends itself well to publishing linked open data, with URLs representing entities rather than actions, and the system uses content negotiation approaches [13] to allow each URL in the system to be served via a HTTP GET request as XHTML (for humans) and RDF/XML or JSON for software agents.

Table 1. Noun-based vs. verb-based URL Structure

\begin{tabular}{|l|l|}
\hline URL Structure & \\
\hline Noun-based & $\underline{\text { http://lists.lib.plymouth.ac.uk/lists/abf203 }}$ \\
\hline Verb-based & $\underline{\text { http://example.org/view?courseCode=ABF203 }}$ \\
\hline
\end{tabular}

In the linked data world, agents can follow the dereferencable URIs in one document to another if they wish to augment the description of a particular resource. This can rapidly become unwieldy if documents contain large quantities of links - agents may need to make a large amount of additional HTTP calls for even basic use cases. In line with designing for appropriation, the system makes a reasonable estimate of shape of data that a user agent might expect when requesting a description of a particular class of resource. For example, a GET issued against the URI of a list will also return details of the items in the list.

In general, using a linked data approach makes it very easy for system integrators to either simply link to the system's human-consumable interpretation of the user interface (via the dereferencable URI), or create their own interpretation within their respective VLE or institutional portal environments.

Other services on the web can link to resources described within the system to augment their own descriptions, and mine rich relationships to other related topics or resources.

\subsection{Licensing of Data}

An oft-overlooked aspect of publishing data on the web is the inclusion of specific license terms on what rights the owner of the data set is willing either to exert or waive [17]. As the linked open data web expands, the importance of attaching specific licensing terms will become as important as it has in the open source software world. Knowing that you are free to incorporate a dataset within your own will become as important and knowing that you cannot. The Open Data Commons Public Domain Dedication and Licence ${ }^{14}$ is available for publishers of data to explicitly gift data to the commons according to community norms.

Talis is consulting with customers of the RLM tool to make sure they understand the implications of publishing data on the open web, and encourages them to do so

\footnotetext{
${ }^{14} \mathrm{http} / / /$ www.opendatacommons.org/odc-public-domain-dedication-and-licence/
} 
with explicit license terms. Part of the implementation process involves the selection of an appropriate strategy according to the specific customer's requirements. To encourage the widespread re-use and linking of data, Talis is offering savings on product subscriptions for those customers who select open licensing strategies for their data.

\section{Conclusions and Future Work}

By using existing ontologies and publishing the data within the RLM as linked open data, the tool not only unifies the description of learning resources, but it also unlocks silos of semantically rich descriptions of inter-related resources and the links and relationships between them, as described by domain experts (the instructors). This enables both more effective linking strategies and better interoperability of data between enterprise systems compared to existing systems.

The Talis RLM tool encourages user-enriched descriptions of the data it manages by increasing the opportunities for users to annotate existing data. The aggregate of these enrichments form the basis for explicit feedback to the list creators.

Having unified the descriptions of lists, added semantic richness to the data and encouraged mass user annotations, future development will focus on the issues around context-aware recommendation systems. The first iteration of such a system will be implemented at the point at which the instructor harvests items into the system. Because the system recognises resources not just web links, it can make well informed suggestions based on context rather than keywords. For example, the system may suggest an alternative, newer edition of a book available from library stock, rather than the older copy the instructor is harvesting from Amazon.

This richness in description, allowing the identification of similar or equivalent items, can also be applied to the problem of identifying patterns in stock usage. Combined with the collective intentions of the user base, gleaned from annotations made by students as to their likely use of the item, customers can predict and plan an optimal stock strategy more accurately than in a system that relies on proprietary identifiers alone.

At the time of writing, Talis are continuing the process of rolling the system out to further focus group partners, and will continue to implement the system across the existing RLM customer base of 1/3rd of UK Universities (representing over 800,000 students and over 20,000 instructors) through 2009. Published as linked open data, this will represent a significant corpus of discoverable data about how instructors use resources for teaching.

\section{References}

1. World-Wide Web Consortium: Semantic Web Layer Cake (2007), http: / /www.w3 .org/2007/03/layerCake.svg (accessed December 12, 2008)

2. Berners-Lee, T.: Linked Data (2006), http: / /www.w3 .org/DesignIssues /LinkedData.html

3. O'Reilly, T.: What Is Web 2.0 (2005), http://www.oreillynet.com/pub/a/ oreilly/tim/news/2005/09/30/what-is-web-20.html (accessed December $12,2008)$ 
4. Bruffee, K.: Collaborative Learning: Higher Education, Interdependence, and the Authority of Knowledge. Johns Hopkins University Press (1993)

5. Breeding, M.: The Many Facets of Managing Electronic Resources. In: Proc. Computer in Libraries Conference (2004)

6. Shabir, N., Styles, R.: Resource List Ontology, http://vocab.org/resourcelist/schema-20080519.html (accessed December 15, 2008)

7. Leavesley, J. and Davis, I.: Talis Platform: Harnessing Sophisticated Mass Collaboration on a Global Scale, http: / /www.talis.com/platform/resources/assets/ harnessing_sophisticated_mass.pdf (accessed December 12, 2008)

8. Darkus, B.: Bibliographic Ontology, http: //purl.org/ontology/bibo/ (accessed December 15, 2008)

9. Adida, B., Birbeck, M.: RDFa Primer, http: / /www.w3 .org/TR/xhtml-rafa-primer/ (accessed December 15, 2008)

10. Talis Group Limited: Changeset Protocol, http://n2.talis.com/wiki/Changeset_Protocol (accessed December 15, 2008)

11. Brickley, D., Miller, L.: FOAF Ontology, http: / / xmlns.com/foaf / spec/ (accessed December 16, 2008)

12. Miles, A., Brickley, D.: SKOS Core Guide, http: / / www.w3 .org/TR/2005/WD-swbp-skos-core-guide-20050510/ (accessed December 16, 2008)

13. Dublin Core Metadata Initiative: XML Schemas to support the Guidelines for implementing Dublin Core in XML,

http: / / dublincore.org/schemas / xmls / (accessed December 16, 2008)

14. Shabir, N., Styles, R.: Academic Institution Internal Structure Ontology, http: / /vocab.org/aiiso/schema-20080514 (accessed December 15, 2008)

15. Bizer, C., Cyganiak, R., Heath, T.: How to Publish Linked Data on the Web (2007), http://sites.wiwiss.fu-berlin.de/suhl/bizer/pub/ LinkedDataTutorial/

16. Styles, N.: Pages, Screens, MVC and Not Getting It, http : / dynamicorange.com/ 2008/10/03/pages-screens-mvc-and-not-getting-it/ (accessed December $12,2008)$

17. Miller, P., Styles, R., Heath, T.: Open Data Commons, a License for Open Data. In: Proceedings of the 1st International Workshop on Linked Data on the Web (LDOW), 17th International World Wide Web Conference, Beijing, China (April 2008) 